\section{SUPERFICIES FLAMEANTES}

REVESTIMIENTO DUNA DE CASTELATTO PISOS E REVESTIMENTOS VENTA DIRECTA DESDE BRASIL www.castelatto.com.br

Ricardo Mangold

ricardomangold@castelatto.

com.br

sac@castelatto.com.br Teléfono: (55) (11) 44167691 Rua Antonio da Cunha Leite 3070, Bairro do Portão, Atibaia/ São Paulo, Brasil
Los revestimientos cerámicos de la línea Duna responden, mediante su forma innovadora, a las nuevas necesidades de personalización en los proyectos de interiores de arquitectura. A partir de palmetas de concreto de $50 \times 75 \mathrm{~cm}$ o $20 \times 20 \mathrm{~cm}$, este revestimiento consigue un aspecto continuo y particular en toda la superficie cubierta.

Duna es adecuado para todo tipo de muros -interiores y exteriores- y está disponible en diferentes colores, gama de grises, sepia, arena y blanco, entre otros. A través del uso de distintas variedades de piedras en el proceso de fabricación consigue diferentes texturas; tanto un aspecto más rugoso y variado en la Línea Crystalli, o bien una terminación de textura lisa y suave en la Línea Paris.

Se recomienda que su instalación sea hecha con doble capa de mortero flexible, con la posibilidad de dejar una mínima cantería entre las palmetas, o bien la ausencia de éstas.

\section{DATOS TÉCNICOS RELEVANTES}

Formato: palmetas de $50 \times 75 \mathrm{~cm}$ y $20 \times 20 \mathrm{~cm}$

Materialidad: concreto

Colores: gama de grises, sepia, arena y blanco, entre otros

Aplicaciones: uso exclusivo como revestimiento de muros

\section{ILUMINACIÓN ANTIDESLUMBRAMIENTO}

LUMINARIAS CLINIC DE DILAMPSA DISTRIBUIDOR EN CHILE LAMP CHILE www.lamp.es

Cristián Tobar / chile@lamp es Teléfono: (56) (2) 2371770

Exequiel Fernández 2251, Macul, Santiago, Chile

Las luminarias Clinic de D I LA M P S a aportan una solución eficiente, funcional y sostenible para las habitaciones de recintos hospitalarios. Su diseño simple y su estética neutra y poco agresiva permiten evitar el deslumbramiento y el estrés visual.

El sistema de iluminación Clinic para cabezal de cama desaparece del espacio, cede todo el protagonismo a la luz emitida y entrega las herramientas para controlar el deslumbramiento directo y cuidar, por ejemplo, que el paciente no esté expuesto a luminancias mayores de $750 \mathrm{~cd} / \mathrm{m} 2$, mediante un sistema accesible y de fácil control.

Fabricadas en extrusión de aluminio lacado en color blanco, permiten ser personalizadas por medio de la elección de la fuente de luz y sus mecanismos necesarios -interruptores, salidas de teléfono y otros-. Se puede utilizar fuentes de $1 \times 24 \mathrm{~W}$ para luz directa y fuentes de1x54W para luz indirecta.

Una de sus aplicaciones destacadas ha sido la iluminación del nuevo Hospital de Reus en Tarragona, de los arquitectos Corea, Morán y Pich Aguilera, realizado durante el año 2010.

DATOS TÉCNICOS RELEVANTES

Formato: $1270 \mathrm{~mm}$ ó $1860 \mathrm{~mm}$ de longitud de cabezal; $145 \mathrm{~mm}$ de alto y $90 \mathrm{~mm}$ de fondo Materialidad: aluminio lacado

Color: blanco

Aplicaciones: sistema de iluminación para cabezal de camas de hospitales

Variaciones: se puede escoger la longitud del cabezal; el tipo de luz: directa o indirecta; $y$ los mecanismos necesarios para su funcionamiento o elementos complementarios -como interruptores o salidas de teléfono-
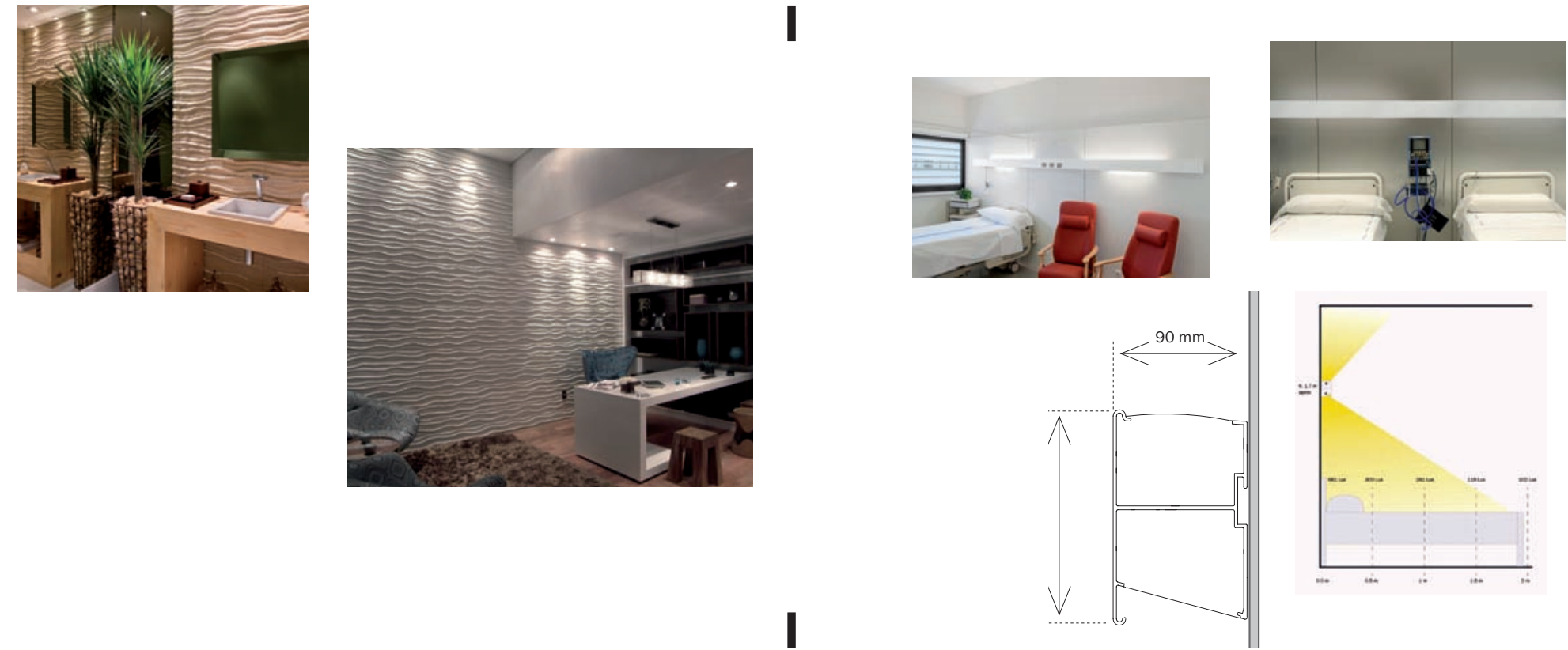


\section{SISTEMAS DE ELEVACIÓN}

SISTEMA DE ELEVACIÓN PARA PUERTAS

AVENTOS DE BLUM

DISTRIBUIDOR EN CHILE

HBT www.hbt.cl

Yael Steckman

ysteckman@hbt.cl

Karina Núñez

knunez@hbt.c

Teléfono: (56) (2) 2017650 anexo

114

Las Hualtatas 7723 , Vitacura,

Santiago, Chile
Los sistemas de elevación para puertas Aventos, ofrecen un programa para diferentes formatos y tipos de aperturas, para distintas materialidades de puertas para muebles de cocina, hogar u oficina.

Ubicados en la parte superior de los muebles, cuentan con un ajuste fino que permite que la puerta se detenga en cualquier posición, cerrando suave y silenciosamente gracias al sistema Blumotion. Aventos puede mejorar su rendimiento y sensación de confort al combinarse con Servo-Drive, un sistema de apertura electro-asistida que se activa a través de un leve toque en el frente del mueble. Es además muy recomendable para modulaciones especiales que, al abrirse, dejan la parte inferior de la puerta muy distante, dificultando el cierre del gabinete.

Al desplegarse, las puertas ofrecen excelente visibilidad y acceso cómodo y ergonómico, que respeta el espacio a la altura de la cabeza.

Aventos está disponible en diferentes versiones: H F para compás abatible plegable, H L para compás abatible vertical, Hs para compás abatible en altura, Н K para compás abatible y $\mathrm{H} \mathrm{K}$ - $\mathrm{S}$ para pequeños compases abatibles.

\section{DATOS TÉCNICOS RELEVANTES}

Materialidad: tecnología Blumotion: conjunto de muelles que garantizan un cierre suave de máxima durabilidad

Color: tapa disponible en blanco

Aplicaciones: muebles murales y módulos aéreos de cocina, hogar y oficina

Garantía: 10 años u 80.000 ciclos de apertura y cierre

Certificaciones: Iso 9001 Certified Quality System e ISo 14001 Certified Enviromental System
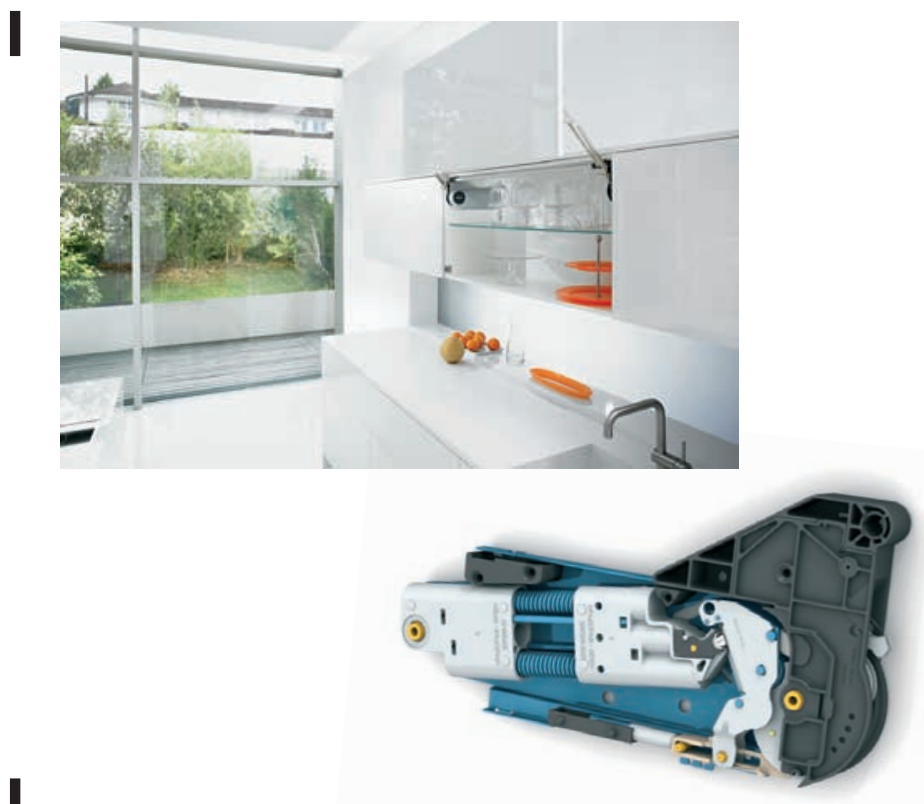

FACHADAS VENTILADAS

PLACAS CERÁMICAS TERRART DE NBK DISTRIBUIDOR EN CHILE

HUNTER DOUGLAS CHILE S.A.

www.hunterdouglas.cl

José Luis López

jlopez@hdlao.com

Teléfono: (56) (2) 3940300 Av. Bicentenario 3883 , oficina 1 Vitacura, Santiago, Chile

NB K es un revestimiento cerámico HUNTER DOUGLAS de gran formato para fachadas ventiladas. La arcilla con la cual se fabrican estos paneles, aporta calidez y durabilidad a las superficies y tiene además la inherente sustentabilidad de este material.

Este sistema trabaja con elementos desarrollados especialmente para cada proyecto. Detrás de las canterías verticales se encuentra la estructura soportante, que permite fijar este revestimiento a la estructura del edificio, mejorando su comportamiento técnico.

Las placas están construidas de tal forma que el aire puede pasar a través de las canterías horizontales abiertas, lo que produce una compensación de la presión del aire entre el espacio ventilado y el aire exterior. Debido al diseño machihembrado de las canterías y la diferencia de presión, las aguas lluvias no penetran en los espacios interiores.

\section{DATOS TÉCNICOS RELEVANTES}

Formato: cerámicos de gran formato $600 \times 1800 \times 40 \mathrm{~mm}$ (espesor); cerámicos de formato mediano 300 x 1200 x 30mm (espesor); cerámicos macizos 600 × 1200 × 30 mm (espesor) Quiebravistas: formas cerámicas especiales, se utilizan comunmente entre 40 x 40 y 50 x $50 \mathrm{~mm}$ de sección transversal y $1.45 \mathrm{~m}$ de largo

Materialidad: cerámico

Colores: gran variedad de colores, es posible realizar cualquier tonalidad

Terminaciones: esmaltada, pulida y natural

Prestaciones: revestimientos exteriores, fachadas ventiladas
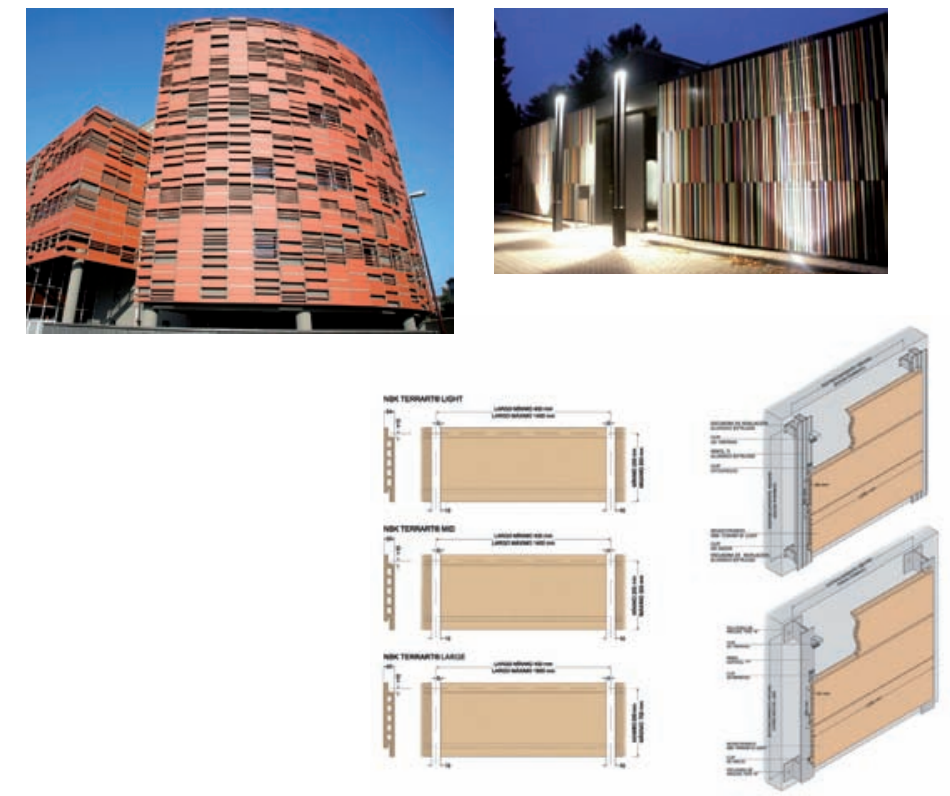


\section{LED EMBUTIDO}

LUMINARIA PL2OW DE LED STUDIO DISTRIBUIDOR EN CHILE LED STUDIO www.ledstudio.c

rriquelme@ledstudio.c

Wilson Valdés

wvaldes@ledstudio.cl

Teléfono: (56) (2) 9823883

Avda. Padre Hurtado 1199 Vitacura,

Santiago, Chile
Las luminarias LED embutidas a cielo PL20W, ofrecen una práctica y eficiente solución para la iluminación interior en obras cuyo programa alberga por ejemplo: malls, locales comerciales, oficinas u otras diversas aplicaciones relacionadas a este tipo de arquitectura interior.

Estos equipos, en su modelo de $20 \mathrm{~W}$, son capaces de homologar perfectamente lámparas tipo PL convencionales, lo que significa un ahorro en los costos de energía y por lo tanto una solución más sustentable.

De fácil instalación, las ventajas de este equipo LED son múltiples: ahorra hasta un $90 \%$ de energía, tiene una vida útil sobre las 50.000 horas, alta resistencia a impactos, no genera calor, no contiene mercurio y no requiere mantención.

Adicionalmente, el diseño simple y limpio de este equipo lo hace muy versátil, lo que permite su aplicación en una diversidad de espacios.

\section{DATOS TÉCNICOS RELEVANTES}

Formato: $204 \mathrm{~mm}$ de frente, $115 \mathrm{~mm}$ de fondo y $226 \mathrm{~mm}$ de diámetro en la parte embutida Materialidad: aluminio de alta resistencia

Colores: blanco, frío o cálido

Consumo: $20 \mathrm{~W}$

Voltaje: $220 \mathrm{~V}$

Lúmenes: 1500

Temperatura de trabajo: entre $-20^{\circ}$ y $45^{\circ} \mathrm{C}$

Ángulo: $120^{\circ}$

Prestaciones: locales comerciales, oficinas o aplicaciones similares
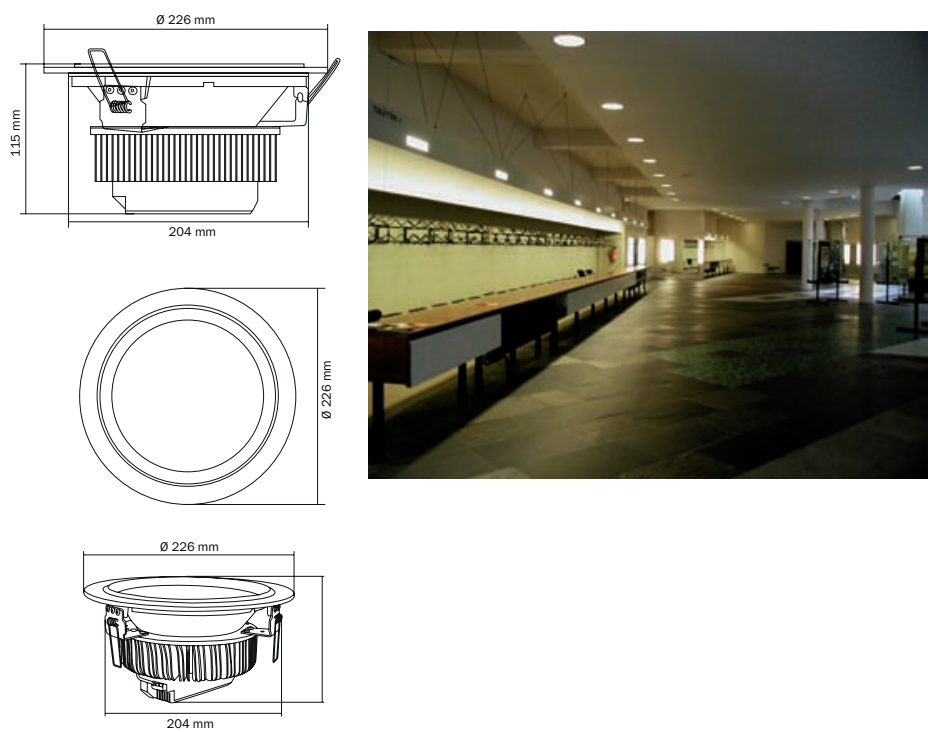

CORTINAS CONTROLABLES

ROLLER QUANTUM DE LUXAFLEX

DISTRIBUIDOR EN CHILE

HUNTER DOUGLAS CHILE S.A. www.luxaflex.cl

Felipe Prieto

fprieto@hdlao.com

Teléfono: (56) (2) 3940000

Avda. Bicentenario 3883, Vitacura

Santiago, Chile

El mecanismo para las cortinas interiores Roller Quantum, disponible en las líneas Q31, Q37 y Q50 -representando estos números el diámetro interior de cada tubo en milímetros- y en cuatro tipos de terminación: Standard, Perfil Plus, Cenefa frontal y Cenefa completa, posee un nuevo e innovador sistema de control que proporciona un acondicionamiento liviano y preciso a los espacios. Dentro de las innovaciones sobre este diseño están los nuevos encajes del sistema de control al soporte, que otorgan mayor seguridad en la instalación, así como una puntera provista de un resorte que puede extender la cortina corrigiendo errores de medición de hasta $14 \mathrm{~mm}$, sin poner en riesgo la seguridad. Tiene además distintas alternativas de bases: ovalada (que puede ser a la vista o forrada con tela), slim y una especial para su uso con perfiles laterales y telas black-out.

Opcionalmente para estos modelos es posible incorporar, al costado opuesto del sistema de control, un conjunto nivelador que posibilita la estabilidad con la cortina ya instalada, en un rango -hacia para arriba o hacia abajo- de aproximadamente $4 \mathrm{~mm}$. También es útil para evitar problemas de roce lateral de la tela.

\section{DATOS TÉCNICOS RELEVANTES}

Formato: altura máxima, según colección, entre $1300 \mathrm{~mm}$ y $3000 \mathrm{~mm}$. Q31: $31 \mathrm{~mm}$ de diámetro interior del rollo; Q37: $31 \mathrm{~mm}$ de diámetro interior del rollo; Q50: $50 \mathrm{~mm}$ de diámetro interior del rollo

Profundidad mínima de vano: entre 65 y $85 \mathrm{~mm}$, según soporte

Materialidad: piezas del soporte de aluminio y acero inoxidable

Telas disponibles: en base a trevira, fibra de vidrio con PVC, poliéster y poliéster con PVC. Además de telas naturales en base a jute, celulosa y algodón

Colores componentes: blanco, gris y negro

Telas: amplia gama de tonos

Terminación: diferentes tipos de soportes, cenefas y bases

Prestaciones: control y protección solar de interiores.
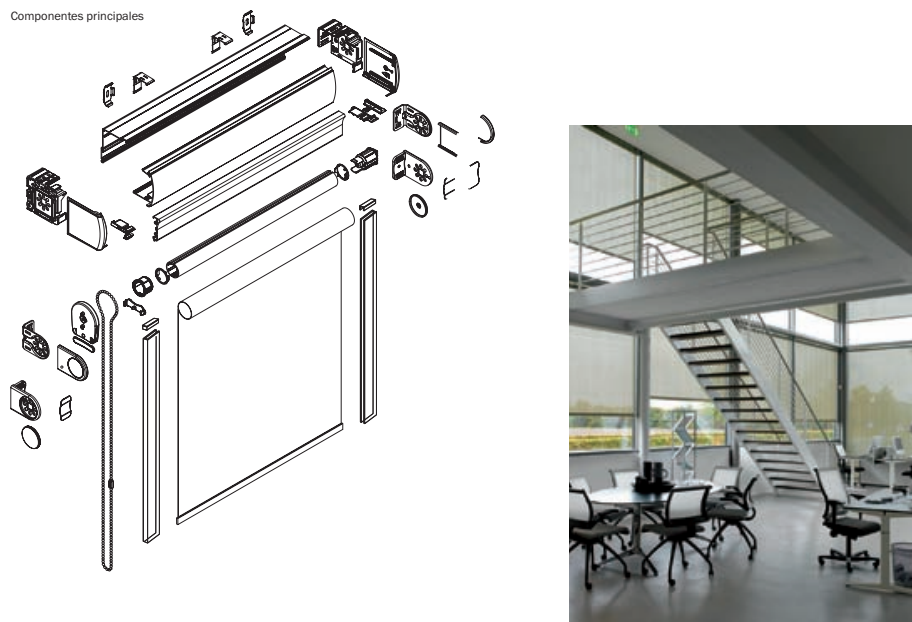


\section{FACHADA FIBROCEMENTO}

SWISSPEARL DE ETERNIT SUIZA

DISTRIBUIDOR EN CHILE

COMINTECC SUIZANDINA www.comintecc.cl/swisspearl.

html

Angélica Ramírez

aramirez@comintecc.com

Teléfono: (56) (2) 2023107

Las Tranqueras 1395, Vitacura,

Santiago, Chile

\section{CONTROL SOLAR}

CELOSÍAS LÍNEA TRESPA METEON DE

TRESPA

DISTRIBUIDOR EN CHILE

TRESPA CHILE LTDA. www.trespa.com

cl, www.trespa.info Rodrigo Gana

infochile@trespa.com

Teléfono: (56) (2) 4410618 Vitacura 2939 piso 10, Vitacura,

Santiago, Chile

El sistema de fachadas ventiladas Swisspearl está elaborado con paneles de fibrocemento de tecnología suiza, desarrollada sin amianto desde 1990. Esta estructura de revestimiento, ofrece una capa exterior duradera, no inflamable ni dañina para el medio ambiente y con alta resistencia a condiciones adversas a la intemperie. La estructura también contribuye con un sistema de ventilación posterior, que elimina la humedad y optimiza la eficiencia de la aislación térmica de la envolvente del edificio.

Disponible en gran cantidad de colores, permite revestir con una amplia libertad creativa diversos tipos de proyectos y contribuye al desarrollo de soluciones constructivas y terminaciones.

Swisspearl es fácil y rápido de instalar durante cualquier estación del año, mediante un sistema de fijación para sub-estructuras de aluminio, acero y madera. No requiere de sellos en las uniones lo que brinda terminaciones que no necesitarán mantenimiento $\mathrm{y}$, al no tener puentes térmicos, aportarán al confort térmico-acústico de la obra.

\section{DATOS TÉCNICOS RELEVANTES}

Formato: 3,04 x 1,22 $\mathrm{m}$ y $2,5 \times 1,22 \mathrm{~m}$

Espesor: $8 \mathrm{~mm}$

Peso: $16 \mathrm{~kg} / \mathrm{m}^{2}$ aproximadamente

Materialidad: $40 \%$ de cemento Portland, $11 \%$ arena, $2 \%$ de fibras de refuerzo de polivinyl

alcohol, $5 \%$ de fibras de celulosa, $12 \%$ de agua, $30 \%$ de aire

Colores: disponible en más de 75 colores

Aplicaciones: para todo tipo de construcciones, tanto en exteriores como interiores

Conductividad térmica: $0,56 \mathrm{~W} / \mathrm{m}^{\circ} \mathrm{C}$

Certificaciones: resistencia al fuego $\mathrm{A} 1$

Detalles encuentro ventana

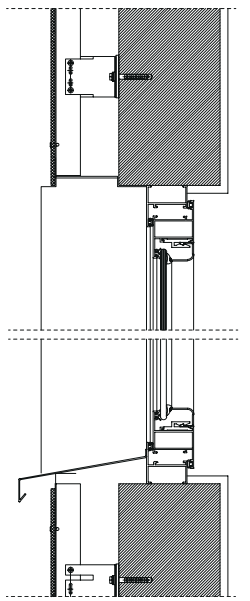

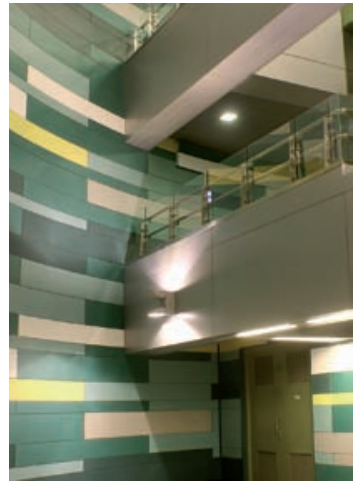

Las celosías de la Línea Trespa Meteon para el control solar, generan una segunda piel en el exterior de los edificios, lo que reduce la carga térmica de estos y permite el aprovechamiento de la luz natural y una correcta ventilación, sin obstaculizar las vistas hacia el exterior.

Como aporte a las estrategias bioclimáticas de los proyectos de arquitectura, las celosías pueden ser diseñadas en diferentes direcciones, dependiendo de la orientación de la fachada, así como también pueden estar compuestas de diafragmas fijos o móviles.

Como acabado, es posible escoger placas con terminación por una o las dos caras, así como la inclusión de perforaciones de diferente geometría, realizadas con máquinas de tecnología $\mathrm{CNC}$. Se ofrecen además alternativas de terminación para las esquinas.

Su materialidad, lograda a base de resinas termoendurecibles, es de alta densidad y por lo tanto de gran resistencia química y estructural, tanto a la humedad como a los rayos Uv. Su garantía de invariabilidad del color es por 10 años, lo que se traduce en bajos costos de mantención.

\section{DATOS TÉCNICOS RELEVANTES}

Formato: dos formatos base de 3650 x $1860 \mathrm{~mm}$ y 4270 x $2130 \mathrm{~mm}$; espesores de 8,10 y $13 \mathrm{~mm}$

Materialidad: $30 \%$ resinas especiales termoendurecibles y $70 \%$ fibras de madera Colores: 62 colores lisos, 13 metálicos, 6 patinados y 17 simulaciones de madera Texturas: Satín, Rock y Gloss

Prestaciones: fachadas ventiladas y tradicionales expuestas a condiciones ambientales adversas, además de pantallas de control solar

Certificaciones: IS09001 y IS014001; European Union of Agrement (Torroja, Komo, DIBT, BUTGB, BBA y CSTB); reconocimientos municipales de Nueva York (MEA) y Miami (Dade NOA)

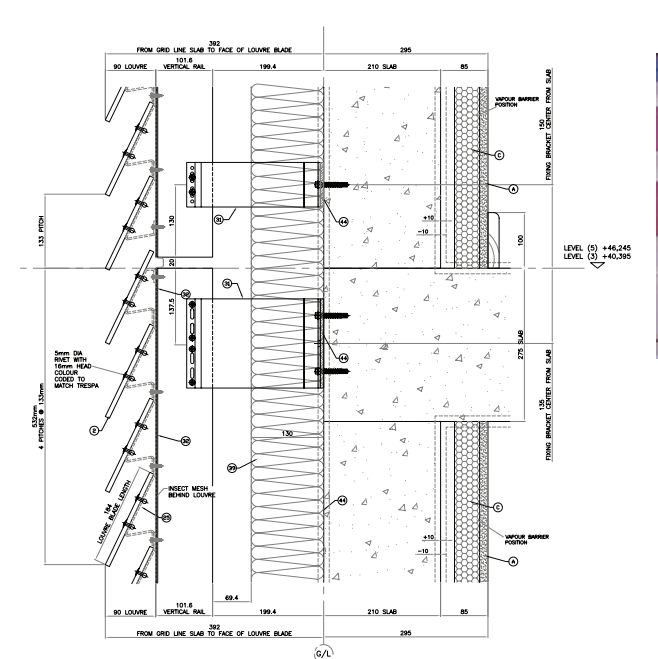

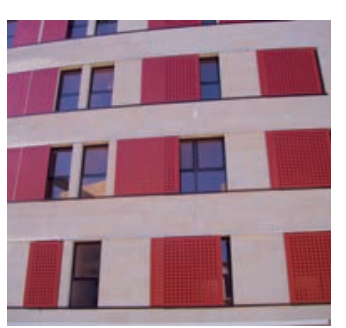

